\title{
LA NARRATIVA D'AMORE E LA CENSURA A BISANZIO
}

\author{
Nota del m.e. FABRIZIO CONCA (*)
}

(Adunanza del 26 marzo 2015)

SunTo. - Il patriarca bizantino Fozio nella Biblioteca esprime un giudizio molto severo sul romanzo di Achille Tazio, Leucippe e Clitofonte, per l'oscenità del contenuto e del linguaggio; lo conferma in questo lavoro l'analisi di alcuni passi significativi, che dimostrano come al Libesroman non siano estranee tematiche licenziose Ma nonostante la "censura" di Fozio, il romanzo ebbe una notevole fortuna in età bizantina, giustificata anche dal fatto che secondo il Lessico Suida Achille Tazio alla fine della vita divenne cristiano e vescovo.

$$
* * *
$$

ABSTRACT. - In his Bibliotheca, the Byzantine patriach Photius is very critical about Achilles Tatius' novel, expressing a very harsh judgment because of the obscenity of the content and the indecency of the language. In the present paper this is confirmed by the analysis of significant passages, which demonstrate how licentious themes are not absent from the Libesroman. Despite of Photius' censorship, the novel enjoyed a remarkable popularity during the Byzantine age, that was also legitimized by the assumption of the Suida Lexicon that Achilles Tatius became a Christian and a bishop towards the end of his life.

La licenziosità del linguaggio e delle scene è sicuramente un tratto rilevante della narrativa d'amore di tradizione greca, che non si esaurisce nello stereotipo amore e avventura, vissuti in una sequenza di peripezie in cui la coppia protagonista mantiene, nonostante molteplici minacce, castità e fedeltà. Sotto tale punto di vista forniscono una testi-

(*) Università degli Studi di Milano, Italia. E-mail: fabrizio.conca@unimi.it 
monianza significativa i frammenti dei Phoinikika di Lolliano - autore oscuro, forse solo omonimo del famoso sofista di Efeso, vissuto al tempo di Adriano e di Antonino Pio.'

In un passo del papiro di Colonia che tramanda il romanzo (fr. A 2 recto, 8-14) è conservata una scena sorprendente in cui l'io narrante è un uomo, che rivela la propria prima esperienza d'amore con una donna, Perside, verosimilmente una protagonista $(« \mathrm{E}$ allora io provai per la prima volta l'amore; e per questo lei prese e voleva darmi i gioielli che indossava, come compenso per la perdita della verginità. Ma io dissi che non li accettavo. Allora lei chiama a sé Glaucete e, quando arriva, li dà a lui e gli ordina di darli al tesoriere e di computare duemila dracme da parte loro. ${ }^{2}$ Poi si girò su di me e non smise fino a che ci prese entrambi (la sazietà) e spuntò il giorno» ${ }^{3}$ ). Dunque, un uomo racconta di avere perso la propria verginità e significativamente nel testo ricorre

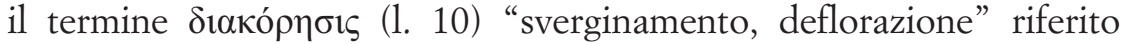
all'uomo e non alla donna, come di consueto, creando un tocco di bumour autoironico, ${ }^{4}$ sottolineato dal fatto che la scena sembra svolgersi in un bordello gestito da un cassiere al quale Perside versa denaro per avere "iniziato" il giovane parthenos - con un palese rovesciamento del topos della "prima volta".

Una scena orgiastica contrassegnata da forte erotismo è testimoniata anche da un altro passo dei Phoinikika (fr. B 1 verso, 18-21): «Dopo che tutti furono arrivati e nessuno era più fuori, chiuse le porte, cantavano, bevevano, si univano alle donne sotto lo sguardo di [». Quale che sia la scelta testuale per colmare la lacuna, ${ }^{5}$ assistiamo a una scena «scioccante perché infrange il comune senso del pudore», ben lontani dal tono "idealizzato" che di solito attribuiamo al Liebesroman.

In verità, gli effetti prodotti dalle letture di storie romanzesche non sembrano sfuggire neppure a Giuliano, Ep. 89b, 300c-301ac:

1 Sull'oscura identità dell'autore, cfr. Henrichs 1972, 24-27 - edizione di riferimento basata su P. Colon. Inv. 3328.

2 Il corsivo evidenzia $\pi \alpha \rho$ ' $\alpha \dot{v} \tau[\tilde{\omega} v]$ lettura di Casanova, in luogo di $\pi \alpha \rho$ ' $\alpha \dot{v}$

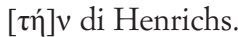

3 Riporto qui e in seguito la traduzione di Casanova 2010, 125.

4 Casanova 2010, 126.

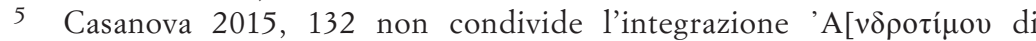
Henrichs. 
I sacerdoti ${ }^{6}$ devono essere immuni non solo dal compiere azioni impure o gesti licenziosi, ma anche dal dire e dall'ascoltare discorsi di tal genere. Dobbiamo eliminare, dunque, ogni facezia inopportuna e ogni conversazione licenziosa. [...] E opportuno che noi leggiamo opere di storia scritte su fatti reali; dobbiamo respingere tutte le favole riferite come storia presso gli antichi, come i racconti d'amore, ed in generale tutte le narrazioni simili. [...] I racconti producono nell'anima uno stato particolare e risvegliano a poco a poco le passioni, quindi d'un tratto accendono una fiamma violenta contro la quale, io penso, bisogna predisporre la difesa da lontano. ${ }^{7}$

a conferma che il romanzo d'amore d'età tardo antica (Caritone, Achille Tazio, Senofonte Efesio, Longo sofista, Eliodoro) circolò con successo e si diffuse anche nella cultura bizantina.

Infatti, nella Biblioteca, Fozio, patriarca di Costantinopoli (858867 e 877-886), riferisce (cod. 87) di avere letto

gli otto libri sulle Avventure di Leucippe e Clitofonte, scritti da Achille Tazio di Alessandria: l'opera è un romanzo che ci presenta vicende d'amore inusitate. Mi pare che questo lavoro si segnali sia per lo stile sia per la struttura: il linguaggio, infatti, è limpido e l'uso dei traslati - ogni volta che l'autore vi ricorre - appropriato; i periodi sono quasi sempre concisi, chiari e gradevoli, e con la loro musicalità seducono l'ascoltatore. Tuttavia, il carattere oltremodo indecente e scabroso delle situazioni rovina da cima a fondo le intenzioni e l'impegno dello scrittore, e induce i lettori a respingere e fuggire tale opera. Questo romanzo - a parte i nomi dei personaggi e l'esecrabile volgarità - presenta una forte somiglianza con le Etiopiche di Eliodoro nella struttura e nell'invenzione dei racconti. ${ }^{8}$

6 Sc. pagani, dal momento che l'epistola ha per tema la restaurazione e la riforma del culto pagano.

7 La traduzione è di Caltabiano 1991, 194-195. Ma in senso opposto, vale la pena anche solo accennare che non molto tempo dopo Teodoro Prisciano (ca.V sec.), archiatra imperiale, nella sua opera Evं ó$_{\rho} l \sigma \tau \alpha$ «Rimedi di uso comune», di cui è conservata solo la traduzione latina fatta dall'autore stesso, raccomandava la lettura dei romanzi di Giamblico e degli autori a lui affini per curare l'impotenza. Anche Paolo di Nicea, autore di oscura identificazione, vissuto dopo il secolo VII d.C., consigliava analoga cura, cfr. Ieraci Bio 1996, 167-168 (c. 81).

8 Qui e in seguito le traduzioni dalla Biblioteca sono di Bevegni in Fozio 1992, 176-177; l'osservazione finale «è in parte inesatta: il romanzo di Achille Tazio si differenzia dalle Etiopiche per il fatto che consiste in una narrazione in prima persona» $(177$, n. 3$)$. 
La Biblioteca testimonia che Fozio conosceva anche romanzi per noi perduti o conservati solo in frammenti: è il caso dei Babyloniaka di Giamblico (cod. 94)

un romanzo che mette in scena una storia d'amore. Egli esibisce un linguaggio meno osceno di Achille Tazio, ma fa mostra di una maggiore impudicizia rispetto al fenicio Eliodoro: questi autori, infatti, si sono tutti e tre proposti in pratica lo stesso scopo - ossia rappresentare avventure di tema amoroso - ma Eliodoro usa un linguaggio più austero e castigato, Giamblico ha meno ritegno di lui, mentre Achille è triviale e indecente oltre ogni limite.

Dunque, il bersaglio del patriarca è Achille Tazio; ma tale condanna «può venir riguardata come un giudizio dell'ortodossia oppure si tratta di un'opinione personale di Fozio?», questo si domanda Hans-Georg Beck, il quale aggiunge che «si tratta certamente della voce di un uomo ortodosso e generalizzando si potrebbe dire, sulla base dell'importanza di Fozio, della voce della coscienza della classe colta a Bisanzio», ${ }^{9}$ in un contesto storico in cui l'ortodossia rappresentò sempre un baluardo sicuro per l'ideologia imperiale, in un momento storico di grandi trasformazioni.

A partire dalla metà del VII secolo gli arabi conquistarono province assai importanti come Egitto, Palestina e Siria e la stessa chiesa imperiale subì notevoli limitazioni territoriali, ma questo non intaccò l'ortodossia che divenne il contrassegno dell'integrazione nell'ideologia imperiale. Lo Stato, in seguito alla perdita d'importanti risorse nelle province cedute, si vede costretto a dipendere dall'aiuto finanziario della Chiesa che, ben conscia della mutata situazione, ne sfrutta l'arrendevolezza nei suoi confronti, imponendo con più energia rispetto al passato i propri orientamenti etici. Emblematico di questa evoluzione è il fatto che il Concilio in Trullo (691-692) convocato da Giustiniano II colpisce con l'anatema tutti i divertimenti popolari che potevano avere un contenuto erotico. ${ }^{10}$

Nel caso specifico di Achille Tazio, alla luce del giudizio di Fozio, ciò che appare biasimevole è l'insistenza didattica del racconto ${ }^{11}$ in

9 Beck 1986, 120.

10 Beck 1986, 114-115.

11 Beck 1986, 117-118 «Una storia d'amore, così viene detto già nell'introduzione [sc. del romanzo], rappresenterebbe un buono stimolo per il desiderio e se qualcuno si è imposto come regola la castità il romanzo lo farà ricredere». 
molte parti infatti la storia diventa una sorta di manuale d'amore in cui il romanziere non trascura alcun dettaglio per spiegare la fenomenologia dell'eros, etero e omosessuale, pur senza intaccare nella sostanza il fondamento irrinunciabile della fabula: i protagonisti potranno godere le gioie dell'amore solo dopo il matrimonio.

L'intransigenza di Fozio aveva dunque fondate ragioni, come cercherò di documentare con qualche esempio. Nel tessuto del romanzo, in cui i mirabilia dell'azione si alternano a excursus di vario argomento (località, animali, opere d'arte, storie di contenuto eziologico e mitico), la didache erotica si coglie in particolare quando i protagonisti cercano di definire i propri comportamenti con riflessioni che rivelano «la tendenza filosofeggiante» ${ }^{12}$ dell'autore.

Così accade in I 10, 2-7 quando Clinia si rivolge al cugino Clitofonte: ${ }^{13}$

Con la tua fanciulla non parlare mai di atto sessuale, ma fai in modo che la cosa avvenga da sé. Ragazzo e ragazza hanno un identico pudore: per quanto siano inclini ai piaceri, non desiderano sentirne parlare, perché ritengono che la vergogna risieda nelle parole. Le donne mature provano piacere anche dalle parole; una fanciulla invece sopporta gli assalti degli amanti portati da lontano e poi d'improvviso, con un cenno, fa capire il suo consenso. Ma se l'aggredisci e chiedi senz'altro la cosa (ह̌pyov), le ferisci le orecchie con la tua voce, arrossisce e rifiuta la proposta, pensando di essere stata infamata; e si vergogna anche solo di promettere il suo favore, perché ritiene di subire l'atto proprio quando prova piacere nell'ascoltare le parole di seduzione. Se invece [...] la rendi ben disposta e ti accosti a lei senza difficoltà, osserva il massimo

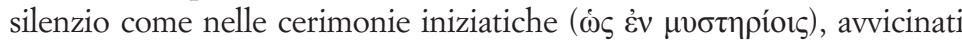
piano piano e baciala, perché il bacio dell'amante per un'amata ormai disposta ad offrirsi è un invito tacito, per una ancora ritrosa è una supplica. Perfino quando è stato raggiunto un patto per passare all'azione

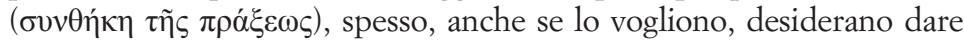
l'impressione di subire violenza, per sottrarsi all'accusa della volontarietà con l'apparenza della costrizione. Dunque, non devi tentennare quando la vedi opporsi, ma considera come lo fa: anche in questo caso bisogna essere abili. Se continua a fare resistenza, astieniti dalla violenza, perché non è ancora convinta; ma se ormai la sua volontà è diventata più fiacca, recita bene la tua parte per non rovinare la tua messinscena.

12 Castiglioni 1923, 30.

13 Ove non indicato, le traduzioni che seguono sono di chi scrive. 
Questi ammaestramenti trovano un completamento nei logoi erotikoi (II 37-38), nei quali alle gioie dell'amore eterosessuale descritte da Clitofonte, sono contrapposti i piaceri dell'eros paidico, prediletto da Menelao, fedele compagno d'avventura della coppia protagonista, in una variegata esplosione verbale che fa comprendere la condanna di Fozio e che non trova esempi nella narrativa greca d'amore della tarda antichità.

Il logos di Clitofonte (II 37) è un inno alla sensualità femminile, colta all'apice dell'amplesso, con un realistico compiacimento che sconfina nel voyeurismo. La tensione dell'atto è scandita da scelte stilistiche che accompagnano con icastica precisione il movimento dei corpi, che trovano un sigillo nei baci; la donna «rende il bacio più dolce perché non si limita a baciare con le labbra, anche i denti contribuiscono al bacio; si nutre della bocca di chi bacia, e morde i baci» $(37,7)$. Il bacio si mescola all'ansimare dell'amplesso, «lo segue, fino a colpire il cuore: il cuore, sconvolto dal bacio, batte forte; e se non fosse legato alle viscere, lo seguirebbe e sarebbe trascinato in su verso i baci. I baci dei ragazzi invece sono rozzi, i loro abbracci senza finezza, i loro atti sessuali fiacchi, nessuna traccia di piacere» $(37,10)$. Significativamente la pulsione dell'atto è enfatizzata sul piano stilistico dall'alternanza dei preverbi/avver-

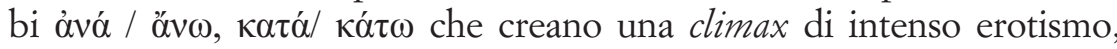
tanto che non sembra esagerato affermare che «non ci è pervenuto nessun passo della letteratura greca in cui la sensualità femminile (o meglio, la pretesa sensualità femminile, dal momento che a parlare è pur sempre un uomo) sia descritta con tanta ampiezza e libertà». ${ }^{14}$

Naturalmente, la conclusione di Clitofonte è una provocazione che stimola la replica di Menelao. Nella donna tutto è artefatto: si fa bella con profumi o tinture, «ma se la privi degli inganni, che sono molti, somiglia alla cornacchia della favola, spogliata delle penne» $(38,2)$. La bellezza dei ragazzi invece «non è irrigata da unguenti e da profumi, o da altri odori ingannevoli ed estranei al loro corpo» (38, 3), e la flessuosità delle carni non rammollisce i corpi, che «offrono resistenza l'uno all'altro, lottando in una gara che ha in palio il piacere» $(38,4)$. Certamente, se l'aischrologhia di Clitofonte non poteva non attirare il biasimo di Fozio, la replica di Menelao appare ancor più traumatica, considerata la durezza con cui era stata colpita la

14 Zanetto 2012, 118. 
pederastia a partire da Giustiniano (Novelle, 77 [535], 141 [559]) e che trova conferma nell'assenza di qualsiasi allusione all'amore paidico pure negli epigrammi del VI secolo.

$\mathrm{Ma}$ anche un altro episodio contribuisce senza dubbio a giustificare la censura di Fozio. Clitofonte crede che Leucippe sia morta; è libero e attira su di sé la passione di una donna Melite, giovane, ricca e bellissima, da poco vedova.

Clitofonte, pur oppresso dal dolore, non appare insensibile e accetta di compiacere Melite, purché «non dia fastidio, costringendomi al rapporto, finché non arriviamo a Efeso. Perché in precedenza ho giurato di non avere rapporti sessuali qui, dove ho perso Leucippe » (V 12, 2-3).

Ma all'improvviso ricompare Tersandro, il marito di Melite; di conseguenza Clitofonte non è più promesso sposo, ma adultero e viene messo in catene. In cella riceve la visita della donna, che sfoga la sua passione: «Mio marito mi odia, lo so, e mi accusa di adulterio, un adulterio senza frutto, un adulterio senza amore, che mi ha procurato solo lo vergogna» (V 25, 5). È consapevole di comportarsi «contro ogni decoro», ma non rinuncia a un'estrema supplica «Via, concediti a me!» (V 26, 13). E Clitofonte cede nella convinzione che quanto stava per accadere «non era più un matrimonio, ma una sorta di medicina per un'anima ammalata. Perciò, lasciai che mi abbracciasse, e mentre lo faceva non mi opponevo» (V 27, 3).

Un momento d'amore improvvisato da Eros e suggellato da un pegno, lo scambio delle vesti, che permette a Clitofonte la fuga dalla cella - e proprio la veste fa pensare al ribaltamento della storia di Giuseppe e la moglie di Potiphar. Rispetto alla Genesi, in questo caso la donna appagata libera il prigioniero e custodisce la sua veste quale segno d'amore, non di bybris, come nel racconto biblico. Melite ottiene quel che desidera, ma riuscirà comunque a salvarsi dall'accusa di adulterio, poiché non ha avuto rapporti sessuali con Clitofonte durante l'assenza di Tersandro - con questa imputazione viene sottoposta alla prova dello Stige (VIII 11, 2).

Naturalmente tanta sottigliezza giuridica non poteva convincere Fozio; l'adulterio era perseguito a Bisanzio e merita di essere richiamata almeno l'Ecloga isaurica, promulgata nel marzo 741 (17, 27 «Se un tale commette adulterio con una donna maritata, deve essere tagliato il naso a lui e all'adultera, perché da tale azione consegue il divorzio, deriva la distruzione dei figli e non è rispettata la parola del Signore il quale inse- 
gna che Dio li ha uniti in una sola carne»). ${ }^{15} \mathrm{E}$ la punizione è ribadita anche dal Procheiros nomos databile agli anni 870-879, durante il regno di Basilio I (39, 45 «Gli adulteri, dopo essere stati bastonati e tosati, abbiano il naso troncato. Coloro che sono stati intermediari tra di loro e hanno collaborato a tale scelleratezza, dopo essere stati bastonati e tosati, siano esiliati per sempre»). ${ }^{16}$

La condanna di Fozio rappresenta significativamente una sorta di testimonianza archetipica anche in età moderna - mi limiterò solo a qualche cenno.

Rispetto ai romanzi in particolare, «la riflessione dei censori e dei moralisti si focalizzò innanzi tutto sull'ampio pubblico di riferimento e su quella che appariva loro la natura specifica della ricezione del testo romanzesco: il fatto cioè che si trattasse di una lettura individuale e perciò tanto più coinvolgente»:; ${ }^{17}$ su questa base, il primo romanzo moderno messo all'indice nel 1744 fu Pamela di Samuel Richardson, secondo il parere espresso dal monaco Giovanni Francesco Caravelli il quale «lo collocò nella categoria dei libri osceni, perché vi si trattava di artes amatorias, in base alla settima regola dell'Indice, che imponeva la condanna di libri trattanti di argomenti lascivi in fatto di religione e di costume». ${ }^{18}$

In verità, la questione dei libri da mettere all'indice era stata affrontata dal Concilio di Trento nel gennaio 1562, distinguendo tra $\mathrm{i}$ libri "eretici" e "lascivi", con l'intento non solo di custodire l'ortodossia, ma anche di salvaguardare i costumi e la moralità dei cristiani. ${ }^{19}$

Una significativa conferma è offerta nel 1590 dall'Indice di Sisto V articolato in ventidue regole, che solo la morte del papa ne impedì l'applicazione. La regola XIV proibiva libri omnes qui res obscenas, lascivas, et amatorias vulgari sermone, etiam eleganter conscripti tractant. Necnon comoediae, tragoediae, et fabellae fictae. A preoccupare era soprattutto il pubblico femminile, attratto dalle storie di "armi e amori"; «le donne leggevano: lo si sapeva e anche lo si temeva. Potevano scegliere tra letture piacevoli - che i moralisti ritenevano cattive letture - e letture "buone", elaborate per loro come contravveleno delle "cattive". [...] Ma fu spe-

\footnotetext{
15 Trad. Matino 2012, 174.

16 Matino 2012, ivi.

17 Delpiano 2007, 128.

18 Delpiano 2007, p. 129.

19 Prosperi 2001, 97.
} 
cialmente la varia congerie di romanzi e novelle a trovare nelle donne gli oggetti e le destinatarie», ${ }^{20}$ come aveva denunciato qualche decennio prima l'umanista valenciano Juan Luis Vives (1492-1540) dimostrando che «era proprio dal mondo degli umanisti e dei letterati militanti che saliva la protesta contro l'incontrollata circolazione dei libri; se ne temevano le minacce di corrosione della moralità tradizionale, attraverso la materia amorosa che trattavano o anche attraverso l'irruzione di un immaginario pagano sempre più di moda $\gg^{21}$

Ma i libri continuarono a circolare, proprio come accadde a Bisanzio per Achille Tazio, al quale nonostante la condanna foziana va riconosciuto un felice Fortleben, come documenta la ricca tradizione manoscritta e il giudizio laudativo di AP IX 203:

Amore amaro, ma vita casta: è ciò che il romanzo di Clitofonte mette in mostra. La vita castissima di Leucippe manda tutti in estasi: come resistette, picchiata, tosata, coperta d'oltraggi e, soprattutto, tre volte morta! Se anche tu vuoi essere virtuoso, amico, non badare agli accessori del quadro, ma cogli in primo luogo il succo della storia: approda alle nozze chi ama castamente. ${ }^{22}$

Il lemma non chiarisce la paternità: Fozio patriarca di Costantinopoli, per altri Leone il Filosofo (790-869 ca.). Poiché è impensabile che Fozio si contraddica in modo così palese, l'epigramma deve essere ragionevolmente assegnato a Leone e interpretato proprio come una risposta al patriarca ${ }^{23}$ da parte di una personalità assai autorevole durante l'umanesimo bizantino, ${ }^{24}$ nel tentativo di riabilitare il romanziere - un'operazione confermata anche dal Lessico Suida (I, 439, Adler), nel quale si afferma che Achille Tazio «alla fine della sua vita fu cristiano e vescovo». Un romanzo d'amore scritto da un vescovo doveva per forza risultare incensurabile e avere fortuna tra i lettori. ${ }^{25}$

20 Prosperi 2001, 78.

21 Prosperi 2001, 81.

22 Trad. di Marzi in Marzi-Conca 2009.

23 Cfr. Conca, in Marzi-Conca 2009, 261, n. 1. L'intento del poeta è di sottolineare il messaggio etico del romanzo; con analoga prospettiva i bizantini lessero le Etiopiche di Eliodoro, cfr. Conca 1994, 10-16.

24 Cfr. Lemerle 1971, 149-176.

25 Significativamente anche Eliodoro secondo la tradizione fu vescovo di Tricca (Tessaglia) e anche le Etiopiche furono lette come messaggio etico; lo prova l'interpre- 


\section{BIBLIOGRAFIA}

Beck 1986. H.-G. Beck, L'eros a Bisanzio, traduzione italiana, Kepos Edizioni, Roma, 1994.

Caltabiano 1991. M. Caltabiano, L'epistolario di Giuliano imperatore. Saggio storico, traduzione, note e testo in appendice, M. D'Auria Editore, Napoli, 1991.

Casanova 2010. A. Casanova, Ambienti e luoghi nei frammenti di Lolliano, in I papiri del romanzo greco, Atti del Convegno Internazionale di Studi, Firenze 11-12 giugno 2009, a c. di G. Bastianini e A. Casanova, Istituto Papirologico «G. Vitelli», Firenze, 2010, 121-137.

Castiglioni 1923. L. Castiglioni, Studi intorno ai romanzieri greci. II Achille Tazio, Byzantinisch-neugriech. Jahbücher 4, 1923, 18-50.

Conca 1994. F. Conca, Il romanzo bizantino del XII secolo, UTET, Torino, 1994.

Delpiano 2007. P. Delpiano, I censori leggono i romanzi, in Testi, forme e usi del libro. Teorie e pratiche di cultura editoriale, a c. di L. Braida e A. Cadioli, Sylvestre Bonnard, Milano, 2007, 117-133.

Fozio 1992. Fozio Biblioteca, a c. di N. Wilson, traduzione di C. Bevegni, Adelphi, Milano, 1992.

Henrichs 1972. A. Henrichs, Die Phoinikika des Lollianos. Fragmente eines neuen griechischen Romans, R. Habelt Verlag, Bonn, 1972.

Ieraci Bio 1996. Paolo di Nicea, Manuale medico, a c. di A. M. Ieraci Bio, Bibliopolis, Napoli, 1996.

Marzi-Conca 2012. Antologia Palatina. Traduzione di M. Marzi, introduzione e note a c. di F. Conca, Libri VIII-XI, vol. II, UTAT, Torino, 2009.

Matino 2012. G. Matino, Lex et scientia iuris. Aspetti della letteratura giuridica in lingua greca, M. D’Auria Editore, Napoli, 2012.

Prosperi 2001. A. Prosperi, Censurare le favole. Il protoromanzo e l'Europa cattolica, in Il romanzo, a c. di F. Moretti, I, Einaudi Editore, Torino, 2001, 71-106.

Zanetto 2012. G. Zanetto, I 'discorsi d'amore' nel romanzo di Achille Tazio, in La retorica greca fra tardo antico ed età bizantina: idee e forme, Convegno Internazionale Napoli, 27-29 ottobre 2011, a c. di U. Criscuolo, M. D’Auria Editore, Napoli, 2012, 113-122.

tazione allegorica del romanzo conservata nel cod. Ven. Marc. gr. 410, di difficile attribuzione (Filagato da Cerami, XII secolo ca., ovvero un anonimo del V sec.), secondo la

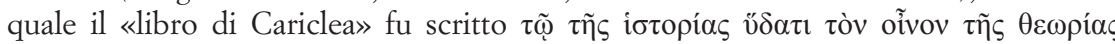
$\kappa \varepsilon \rho \alpha ́ \sigma \alpha \sigma \alpha$. Inoltre, il nome stesso della fanciulla è sinonimo dell'unione tra l'anima e il corpo, nonché di perfezione e rispetto, come recita esplicitamente il testo; lo conferma pure, con un'interpretazione che risente in modo palese delle teorie pitagoriche, il valore numerico delle singole lettere componenti, che sommate danno la cifra 777 . 